\title{
More dialogue, more learning, more action
}

\author{
James Mountford, ${ }^{1}$ Martin Marshall ${ }^{2}$
}

\begin{abstract}
${ }^{1}$ UCLPartners, London, UK
${ }^{2}$ UCLPartners, Lead,

Improvement Science London, London, UK
\end{abstract}

Correspondence to Dr James Mountford, UCLPartners, 3rd floor, 170 Tottenham Court Road, London W1T 7HA, UK; james.mountford@uclpartners. com

Received 9 September 2013 Accepted 9 September 2013 Published Online First 30 September 2013

\section{SLinked}

- http://dx.doi.org/10.1136/ bmjqs-2013-001947

To cite: Mountford J, Marshall M. BMJ Qual Saf 2014:23:89-91.
2013 has not been an easy year for England's National Health Service (NHS). A feeding frenzy led by the popular press exposing deficiencies in care on an almost daily basis, alongside thoughtful challenges from commentators on the 65th birthday (the traditional retirement age in the UK) of what many believe to be 'a national treasure' has led to some serious questions about whether the NHS in its current form is sustainable. ${ }^{12}$ The question is not new, but the intensity of public interest in the quality and safety of care provided by the NHS is breaking new ground, and happening at a time of rising public expectations and decreasing deference across all walks of life.

The publication of a number of seminal reports in 2013 on safety and quality has contributed to this high level of interest. First, in February, a report was published into the tragedy of failings in care at Mid Staffordshire Hospital by Robert Francis, a distinguished barrister. ${ }^{3}$ In its many pages were stories of harrowing gaps in care and compassion, and failure at seemingly every level of the system. Crucially, Francis framed Mid Staffordshire as an extreme example of shortcomings encountered in some form throughout the system. His view was that 'a fundamental culture change is needed', and he made 290 recommendations for how to bring this about. This was followed in July by the publication of a report by Sir Bruce Keogh, the Medical Director of NHS England, into 14 hospitals with the highest mortality rates. ${ }^{4}$ Most recently, August saw the publication of a review into patient safety in the NHS led by Professor Don Berwick, former Centers for Medicare \& Medicaid Services (CMS) administrator and founder of the Boston based Institute for Health Care Improvement, initiated at the personal request of the UK prime minister. ${ }^{5}$

Into this mix, and making a timely and important addition to the literature, steps a paper from Dixon-Woods and colleagues. ${ }^{6}$ The authors report findings from several large studies into quality of care in England's NHS. The ambition and scope of the work is impressive. This is 'meta-research' drawing on multiple different studies, bringing together data from sources as diverse as ethnographic observations and large public health datasets, and using a wide range of social science and other methods to develop new insights into the complex, multifaceted challenge of health system improvement. The approach is ambitious and perhaps, to some methodological purists, contestable. There is a risk that the authors are comparing apples and pears, and such a broad approach may cloud the richness of deeper, more focused analyses. But we think the insights gained by triangulating findings across different studies justifies the authors' approach, and that the results contribute materially to the growing body of knowledge emerging from the recent national reports.

The authors describe how, at a microsystem level, too strong a focus on specific goals and targets can lead to distortion ('hitting the target but missing the point'), and how an overly managerialist perspective can clash with professional norms. Reflecting on organisational performance, they describe how 'bright' and 'dark' spots often coexist side by side within the same organisation, thereby challenging policy makers' propensity to seek single judgments about organisational performance. This chimes with previous findings from USA using quantitative methods comparing mortality rates across hospitals for six common diagnoses which suggested that excellent and poor performance can coexist within one institution, with the department or service being the primary 'axis' around which quality is set. ${ }^{7}$

Dixon-Woods and colleagues introduce a useful insight into how people use data in different ways-'problem seeking' and 
'comfort seeking'-and they describe how innovations in quality improvement are becoming less common as staff respond to increasing workload by battening down the hatches. And they start to probe beneath the surface of the catch-all concept of 'culture', offering a conceptual underpinning for it, and recognising that within any organisation or department, culture is not homogenous, but rather a mosaic of subcultures (often segmenting by profession), all of which contain positive and negative elements which have a rationale and history to them. This sort of deconstruction of culture and its drivers is vital if we are to set about achieving the sort of overarching culture change called for by Francis. ${ }^{8}$

While this research was carried out in one country's health system, many of the conclusions and insights will resonate internationally. So many of the challenges surfaced in this research, and in the Francis, Keogh and Berwick reports, are familiar and shared across health systems whatever their financing, operational structural or governance arrangements may be. Many of the solutions are likely to be common across systems too. The crucial question is: what will it take for this growing jigsaw of empirical research and investigative reports to lead to change at scale and of lasting benefit to patients and populations?

We propose three specific actions which we believe are self-reinforcing and widely applicable to different healthcare settings and organisations. We are working to shape and embed these in our own work at UCLPartners, an academic health science system that brings together NHS providers and commissioners, higher education institutions, communities and local government across North and East London and surrounding counties, covering a population of six million people. ${ }^{9}$ We make no claim that these are the only things that matter, or that they are easy. But we are confident that, if carried out with a strong sense of shared vision and purpose, an ethos of humility and a climate of mutual learning, they will make a difference.

First, is the importance of taking charge of our own destiny and taking action today. We should not simply wait for others to bring about the change we seekwe should try to become more 'playwright' than 'critic'. This is easy to say and feels difficult to deliver, particularly in the context of the reality of delivering care at the front line, where clinicians and managers are often buffeted by events seemingly outside their control. But an unrelenting focus on knowing what it feels like to be a patient, and concentrating on patients' needs within the context of the broader health system can be remarkably enabling. What politicians and system leaders do can make the job of front-line staff harder or easier. But, their ability to force people to do something is limited. On a day-to-day basis, clinicians and managers have greater agency than they realise, or are sometimes willing to accept. Here, Dixon-Woods and colleagues' finding of near-universal commitment from those at the front line to providing safe, high-quality patient-centred services should reassure us that (contrary to what one might conclude from those rare situations where culture becomes toxic) unleashing the front line will lead to greater patient benefit rather than 'capture' where the interests of staff are put ahead of patients'.

Ultimately, whatever our role and position in a health system hierarchy, we can only control our own actions and seek to influence but not dictate what others think and do-and, whatever the circumstances, we always face a choice about how to behave. ${ }^{10}$ Leaders who succeed in facilitating change and maintaining a positive view of their work tend to be those who focus on a small number of locally owned learning and improvement goals, align their immediate colleagues around them, and demonstrate the power of collective action.

Second, is the importance of establishing networks of people with complementary skills and purposefully creating space to think and act differently. The Keogh report identified isolation as a key feature in the aetiology of underperformance. Peer networks (which can be formal and structured, or informal and emergent) can foster a sense of energy, build resilience and provide useful scrutiny, as well as seeding knowledge and innovation. ${ }^{11}$ Within these networks, we can carve out time and space to think and reflect. These are essential elements for effective learning. Day jobs are getting busier and the propensity to be 'always on', enabled by mobile technology, is increasingly shown to be linked to higher stress and reduced effectiveness. $^{12}$

Leaders in other sectors are addressing this by purposefully creating places for people to pause, reflect and talk-and doing so from the realisation that this is necessary for an organisation's on-going success. Traditional linear and instrumentalist approaches to change in organisations based on rational analysis and rigid prescription are being replaced by a more flexible and emergent approach which recognises dialogue as a key part of the improvement, rather than an irritating step along the way. ${ }^{13}$ Too often we feel we don't have time for a cup of coffee with a colleague, but the most enlightening, enduring and productive moments of the day frequently lie in those conversations. We should do our utmost to make dialogue happen, and those responsible for timetabling serve their organisations and staff well when they understand the benefits of doing so. Open conversations, initially unstructured, often lead to the emergence of new ideas, new plans and productive action. ${ }^{14}$

Third, is the importance of developing a new kind of relationship, or 'compact' between clinicians and their employing organisations. There is growing evidence that the compact, or set of commitments that two or more groups in an organisation or system 
make to each other, can be a powerful and enduring tool for improvement. ${ }^{15} 16$ The process of dialogue, enquiry and negotiation by which the compact is agreed is as (or more) important than the content of the compact. Dialogue between groups which may have different norms, values and practices help to force disclosure, build mutual understanding and alignment, and in so doing build relationships and respect. Stereotypical criticisms become tempered: 'the management aren't bad, they're also under pressure and just see the world in a different way'; 'the clinicians aren't trying to be obstructive, they just get frustrated when they have to work around institutional barriers'.

In essence, a compact identifies a set of core commitments each side makes to the other: 'we will do...' and 'in return we expect...'. Compacts can be made at the level of whole organisations or smaller groups within organisations, and have been shown to improve morale and to reshape professional attitudes, even values. Compacts can help articulate why jobs need to change from what people originally thought they signed up to, for example, helping clinicians to understand why their results should be measured more rigorously and reported more transparently, or why a 7 days a week shift pattern is necessary to improve patient access, safety and experience. A willingness to develop a new compact is a practical manifestation of an organisation's commitment to valuing their staff. Across a wide range of industries we know that staff who feel cared for and valued give more to their organisations and their customers. ${ }^{17}$ An important element of the compact, highlighted by the Berwick report, is a willingness on the part of employers and staff to ensure that people have the capability and the capacity to contribute to system-wide improvement.

The common thread through it all is learning. We agree with Berwick that making the NHS a learning system, built up of learning organisations, ${ }^{5}$ is the most powerful and sustainable mechanism for change that we have. The research that Dixon-Woods and colleagues provide is an important substrate for learning to inform conversation and action, and in this lies its importance. Learning together will shape common purpose and an enhanced form of professionalism across the workforce, making us more satisfied and more resilient in our work-and above all more effective in the service of patients and communities.

Competing interests None.
Provenance and peer review Not commissioned; internally peer reviewed.

\section{REFERENCES}

1 Black N. Can England's NHS Survive? N Engl J Med 2013;369:1-3.

2 Nuffield Trust. [17 August 2013]; http://www.nuffieldtrust.org. uk/nhs-65 ?gclid=CP2VquHehLkCFaPHtAodRBwA8A

3 Final Report of the Mid Staffordshire NHS Foundation Trust Public Inquiry (Robert Francis, chair). Staffordshire, UK: Mid Staffordshire NHS Foundation Trust, 2013.

4 Keogh B. Review into the quality of care and treatment provided by 14 hospital trusts in England: overview report. NHS England. London, 2013. http://www.nhs.uk/ NHSEngland/bruce-keogh-review/Pages/published-reports.aspx

5 Berwick D. A promise to learn-a commitment to act: improving the safety of patients in England. Department of Health, London, 2013. https://www.gov.uk/government/ publications/berwick-review-into-patient-safety

6 Dixon-Woods M, Baker R, Charles K, et al. Culture and behaviour in the English National Health Service; overview of lessons from a large multi-method study. BMJ Qual Saf 2014;23:106-115.

7 Rosenthal GE, Shah A, Way LE, et al. Variations in standardized hospital mortality rates for six common medical diagnoses: implications for profiling hospital quality. Med Care 1998;36:955-64.

8 Davies H, Mannion R. Will prescriptions for cultural change improve the NHS? BMJ 2013;346:19-21.

9 Fish D. Academic Health Sciences Networks (AHSNs) in England. Lancet 2013;381:18-9.

10 Frankl VE. Man's search for meaning. Boston, MA: Beacon Press, 2006.

11 Greenhalgh T, Robert G, MacFarlane F, et al. Diffusion of innovations in service organisations: systematic review and recommendations. Milbank Q 2004;82:581-629.

12 Castells M, Fernández-Ardèvol M, Qiu JL, et al. Mobile communication and society: a global perspective. Cambridge, MA: MIT Press, 2006.

13 Bushe GR, Markshak R. Revisioning organization development: diagnostic and dialogic premises and patterns of practice. J Appl Behav Sci 2009;45:348-68.

14 Mintzberg H. Managers not MBAs; a hard look at the soft practice of managing and management development. San Francisco, CA: Berrett-Koehler, 2005.

15 Silversin J, Kornacki MJ. Creating a physician compact that drives group success. Med Group Manage 2002;47:54-62.

16 Edwards N, Kornacki MJ, Silversin J. Unhappy doctors: what are the causes and what can be done? BMJ 2002;324:835-8.

17 Heskett JL, Sasser WE, Wheeler J. The Ownership Quotient: putting the service profit chain to work for unbeatable competitive advantage. Boston, MA: Harvard Business Press, 2008 . 